to the lead investigator were excluded. Face-to-face interviews were conducted, audio-recorded and transcripts analysed using Thematic Analysis.

Results Nine interviews were conducted; 3 families were excluded as they were known to the lead investigator.

None of the children of the interviewees had formal ACP. A large number of themes emerged, which were collapsed into 31 sub themes, 8 major themes and 3 super-ordinate themes. The superordinate themes were: Communication: A Powerful and Essential Tool to Be Used with Care, Impact of LLC: Beyond the Clinic, and Priorities: Putting Families in Control. Deficiencies in communication - both in its content and form - were the most salient and frequent themes that arose, and interviewees reported lasting negative impact. None of the interviewees reported distress needing intervention as a result of participating in the study.

Conclusions Sensitively conducted qualitative research with bereaved families is safe and feasible. Formal ACP and early prognostic discussions are not yet part of routine paediatric practice in the region. Formal ACP and early integration of palliative care are likely to positively impact subjective experiences as well as outcomes such as realisation of preferred PoD. This study was limited by diagnostic heterogeneity and socio-cultural homogeneity of the sample and potential recruitment and recall bias.

Further training of paediatric professionals in communication skills and ACP, and improved access to specialist PPC are recommended. Further research is needed into a. the impact of ACP in different patient groups and b. to professionals' perceptions and barriers.

\section{G371(P) BARRIERS AND FACILITATORS TO PAEDIATRIC SYMPTOM MANAGEMENT AT END-OF-LIFE: RESULTS FROM A SYSTEMATIC REVIEW AND META-ANALYSIS}

${ }^{1} \mathrm{~K}$ Greenfield, ${ }^{1} \mathrm{~S}$ Holley, ${ }^{1} \mathrm{DE}$ Schoth, ${ }^{2,3} \mathrm{E}$ Harrop, ${ }^{4} \mathrm{~J}$ Bayliss, ${ }^{5} \mathrm{RF}$ Howard, ${ }^{6} \mathrm{M}$ Johnson, ${ }^{1,4} \mathrm{C}$ Liossi. 'School of Psychology, The University of Southampton, Southampton, UK; ${ }^{2}$ Helen and Douglas House Hospices, Oxford, UK; ${ }^{3}$ John Raddliffe Hospital, Oxford University Hospitals NHS Trust, Oxford, UK; ${ }^{4}$ The Louis Dundas Centre, Great Ormond Street Hospital for Children NHS FT, London, UK; ${ }^{5}$ Department of Anaesthesia and Pain Medicine, Great Ormond Street Hospital for Children NHS FT, London, UK; ${ }^{6}$ Patient and Public Representative, NIHR Pain and Palliative Care Clinical Studies Group-Children, London, UK

\subsection{6/archdischild-2020-rcpch.318}

Background Managing symptoms, including pain, at end-of-life for infants, children and young people is challenging due to the broad spectrum of life-limiting or life-threatening conditions, and also differing care needs depending on patients' age. The UK National Institute for Health and Care Excellence (NICE) highlights pain management in paediatric palliative care as a research priority. A clearer understanding of these challenges could inform the development of effective interventions.

Aims We aimed to conduct a mixed-methods systematic review and meta-analysis investigating the barriers and facilitators experienced by patients, carers and healthcare professionals managing symptoms in infants, children and young people at end-of-life.

Methods PROSPERO, Cochrane Library, CINAHL, MEDLINE, PsycINFO, Web of Science Core Collection, ProQuest Dissertations \& Theses Database, Evidence Search and OpenGrey were searched from the inception of each database for qualitative, quantitative or mixed-methods studies that included data regarding barriers or facilitators to paediatric end-of-life symptom management from patients, family carers or healthcare professionals. Search terms were derived from a thorough reading of relevant literature and in discussion with a medical librarian. Data extraction, quality appraisal, and narrative thematic synthesis were undertaken for all eligible studies, with meta-analysis also performed where data permitted.

Results We identified 34 quantitative, 19 qualitative and 17 mixed-methods eligible studies of medium-low quality (assessed using GRADE-CERQual). Themes were generated around the barriers and facilitators experienced by family carers (Treatment efficacy, Treatment side-effects, Healthcare professionals' attitudes, Hospice care, Homecare, Families' symptom management strategies) and Healthcare professionals (Medicine access, Treatment efficacy, Healthcare professionals' demographics, Treatment side-effects, Specialist support, Healthcare professionals' training, Health services delivery, Homecare). Only one study included patients' views. Caring at home for a child was reported as a barrier to symptom management by between 22 and 24\% of participants (pooled weighted mean of $0.244, \mathrm{k}=3, \mathrm{n}=197,95 \% \mathrm{CI}=$ 0.177-0.326).

Conclusions This review highlights the need for clear communication within and between healthcare professionals and families; more training for healthcare professionals and improved symptom management planning, including anticipatory prescribing. There is an urgent need for more research into children and young people's experience of symptom management at end-of-life.

\section{G372(P) A SYSTEMATIC REVIEW OF BREAKTHROUGH PAIN ASSESSMENT TOOLS AND THEIR PSYCHOMETRIC PROPERTIES}

${ }^{1} \mathrm{~K}$ Greenfield, ${ }^{1} \mathrm{~S}$ Holley, ${ }^{1} \mathrm{DE}$ Schoth, ${ }^{2,3} \mathrm{E}$ Harrop, ${ }^{4} \mathrm{~J}$ Bayliss, ${ }^{5} \mathrm{~A}-\mathrm{K}$ Anderson, ${ }^{6} \mathrm{RF}$ Howard, ${ }^{1,4} \mathrm{C}$ Liossi, ${ }^{7} \mathrm{M}$ Johnson, ${ }^{4} \mathrm{D}$ Rajapakse, ${ }^{8}$ LK Fraser, ${ }^{2} \mathrm{C}$ Mott, ${ }^{9}$ I Wong. ${ }^{1}$ School of Psychology, University of Southampton, Southampton, UK; ${ }^{2}$ Helen and Douglas House Hospices, Oxford, UK; ${ }^{3}$ John Radcliffe Hospital, Oxford University Hospitals NHS Trust, Oxford, UK; ${ }^{4}$ The Louis Dundas Centre, Great Ormond Street Hospital for Children NHS FT, London, UK; ${ }^{5}$ Royal Marsden Hospital, Sutton, UK; ${ }^{6}$ Department of Anaesthesia and Pain Medicine, Great Ormond Street Hospital for Children NHS FT, London, UK; ${ }^{7}$ Patient and Public Representative; ${ }^{8}$ Martin House Research Centre, University of York, York, UK; ${ }^{9}$ School of Pharmacy, UCL, London, UK

\subsection{6/archdischild-2020-rcpch.319}

Background Breakthrough pain is commonly experienced by both children and adults with cancer, and also by patients with other terminal conditions, including those approaching end-of-life. Management of breakthrough pain is often insufficient, which could be due in part to a current lack of validated assessment tools. The aims of this systematic review are to (1) identify all available instruments measuring breakthrough pain in infants, children, adolescents or adults, and (2) critically appraise, compare and summarise the quality of the measurement properties of each identified instrument using established COSMIN criteria.

Methods Two searches were carried out, one corresponding to each aim of the review. For both, the Cochrane Library, PROSPERO, EMBASE, CINAHL, MEDLINE, PsychINFO, Web of Science Core Collection, Google Scholar, the ProQuest Dissertations \& Theses Database, Evidence Search and OpenGrey were searched from the inception of each database to October 2019. Search terms were derived from a thorough 
reading of relevant literature and in discussion with a medical librarian.

Results Seven breakthrough pain tools were identified, only one of these - The Breakthrough Pain Questionnaire for Children - was designed for use in children. This tool, however, has not been validated to date. A further 40 studies were identified that used bespoke breakthrough pain assessment tools.

Conclusion There is a lack of validated tools for assessing breakthrough pain, particularly in infants, children and young people. These are urgently needed so that the assessment and management of this distressing symptom can be further improved.

\section{G373(P) WHEN PARENTS AND PROFESSIONALS AGREE: AN EXAMPLE OF SHARED DECISION MAKING IN A CASE OF SPINAL MUSCULAR ATROPHY TYPE 1}

${ }^{1} S$ Shahid, ${ }^{1} \mathrm{~N}$ Punjabi, ${ }^{1} \mathrm{~N}$ Sahdev, ${ }^{1} \mathrm{H}$ Khalid, ${ }^{2} \mathrm{U}$ Khalid, ${ }^{1} \mathrm{~K}$ Punjabi, ${ }^{1} \mathrm{~A}$ Gour. ${ }^{1}$ Paediatric Intensive Care Unit, St George's Hospital, London, UK; ${ }^{2}$ University College London, London, UK

10.1136/archdischild-2020-rcpch.320

Introduction The media has covered many cases on when parents disagree with the opinions of healthcare professionals, most notably in the case of Charlie Gard.

This particular case of a female infant with spinal muscular atrophy (SMA) type 1 is an example of when the decisions of parents align with the advice of healthcare professionals and attempts to uncover what factors may have contributed to this cohesion.

SMA is the term given for a group of genetic disorders leading to muscle atrophy and weakness. Type 1 is the most severe phenotype, presenting under 6 months of age and with a $68 \%$ mortality rate within 2 years of life.

Case Synopsis Female infant $\mathrm{M}$ was born at term, with no concerns, to consanguineous parents. At 7 weeks old, her mother took her to the GP with concerns of a lack of limb movement. Examination found multiple neurological abnormalities, including absent rooting reflex, global hypotonia and upgoing Babinski reflexes. $\mathrm{M}$ was thus diagnosed with SMA Type 1.

At 16 weeks old, $M$ was admitted to the Paediatric Intensive Care Unit with respiratory deterioration requiring intubation. M's parents decided that a tracheostomy would make her quality of life worse, even if it did prove lifeprolonging. They opted to palliate on non-invasive ventilation.

Discussion The decision to palliate a patient is burdensome to all involved and is influenced by many factors.

Evidence shows that parents of those with SMA assess success of care not on outcome, but on factors including positive communication with the healthcare team. Furthermore, those who highly rate their experience of this communication have lower levels of long-term grief.

Ensuring parental understanding at each stage is crucial for shared decision-making. The parents of this patient were involved in every step. Their refusal of a tracheostomy despite the potential for added life is not a common decision and as both parental and professional opinions here aligned, reflects the honest and realistic communication between both parties.
Conclusion This case shows how effective communication between the medical team and parents can improve the parental experience of paediatric palliation and help attain shared decision-making.

\section{G374(P) ABSTRACT WITHDRAWN}

\section{G375(P) ABSTRACT WITHDRAWN}

\section{Trainees}

\section{G376 UK PAEDIATRIC TRAINEE TRANSITION TO REGISTRAR SURVEY}

${ }^{1,2} \mathrm{~A}$ Nye, ${ }^{1,2} \mathrm{HS}$ Hodgson, ${ }^{2} \mathrm{~F}$ Finlay. ${ }^{1}$ Paediatrics, Royal United Hospital, Bath, UK; ${ }^{2}$ Community Child Health, Virgin Care Services Ltd, Bath, UK

10.1136/archdischild-2020-rcpch.321

The transition from paediatric level 1 trainee to registrar can be a turbulent time for trainees given the significant 'step-up' in responsibility, variation in preceding clinical experience, and limited opportunities to develop leadership and management skills during level 1 training.

Aims To explore paediatric trainees' experiences of transitioning to registrar at a national level, to identify challenges and training needs during this period.

Methods All UK paediatric trainees working on the middlegrade rota were invited to complete an online survey designed using Survey Monkey over a four-week period between September and October 2019. Questions specifically focused on; experience and confidence in conducting registrar-level tasks, support available to facilitate transition, and views on how trainees could be better prepared and supported during this period.

Results A total of 79 responses were received from 8 deaneries. 10 incomplete responses were excluded. $43 \%$ of respondents did not feel that overall they were adequately prepared for becoming a registrar. Registrar-level tasks that respondents felt most confident about were: supporting junior colleagues and knowing when to call the consultant. Since becoming a registrar, the most challenging aspects of the role identified were leading resuscitations and managing safeguarding cases. With regards to mandatory Level 1 training procedures, respondents were least confident at intubation, and most confident at cannulation.

Regarding interventions intended to facilitate transition: $23 \%$ had stepped-up in the same department where they had spent the previous 6 months working as an SHO; 42\% had done a step-up simulation course; 39\% had supervised actingup experience; and $17 \%$ had done registrar-level clinics. Respondents felt that the most helpful interventions to aid in transition would be: supervised acting-up experience and more exposure to safeguarding cases during level 1 training.

Conclusion Results from our survey suggest that a significant proportion of paediatric trainees do not feel prepared for stepping-up to the paediatric registrar role. It also highlights poor utilisation of learning experiences aimed at developing 